\title{
Egg Microinjection and Efficient Mating for Genome Editing in the Firebrat Thermobia domestica
}

\section{$\operatorname{AUTHOR}(\mathrm{S})$ :}

Ohde, Takahiro; Minemura, Toshinori; Hirose, Eiichi; Daimon, Takaaki

\section{CITATION:}

Ohde, Takahiro ... [et al]. Egg Microinjection and Efficient Mating for Genome Editing in the Firebrat Thermobia domestica. Journal of Visualized Experiments 2020, 164: e61885.

\section{ISSUE DATE:}

2020-10-20

URL:

http://hdl.handle.net/2433/258963

\section{RIGHT:}

Copyright @ 2020 JoVE Journal of Visualized Experiments; 許諾条件に 基づいて掲載しています。 


\title{
Egg Microinjection and Efficient Mating for Genome Editing in the Firebrat Thermobia domestica
}

\author{
Takahiro Ohde ${ }^{1}$, Toshinori Minemura ${ }^{1}$, Eiichi Hirose ${ }^{1}$, Takaaki Daimon ${ }^{1}$ \\ ${ }^{1}$ Department of Applied Biosciences, Graduate School of Agriculture, Kyoto University
}

\section{Corresponding Author}

Takahiro Ohde

ohde.takahiro.4n@kyoto-u.ac.jp

\section{Citation}

Ohde, T., Minemura, T., Hirose, E.,

Daimon, T. Egg Microinjection

and Efficient Mating for Genome

Editing in the Firebrat Thermobia

domestica. J. Vis. Exp. (164), e61885,

doi:10.3791/61885 (2020).

\section{Date Published}

October 20, 2020

\section{DOI}

$10.3791 / 61885$

URL

jove.com/video/61885

\section{Abstract}

The firebrat Thermobia domestica is an ametabolous, wingless species that is suitable for studying the developmental mechanisms of insects that led to their successful evolutionary radiation on the earth. The application of genetic tools such as genome editing is the key to understanding genetic changes that are responsible for evolutionary transitions in an Evo-Devo approach. In this article, we describe our current protocol for generating and maintaining mutant strains of $T$. domestica. We report a dry injection method, as an alternative to the reported wet injection method, that allows us to obtain stably high survival rates in injected embryos. We also report an optimized environment setting to mate adults and obtain subsequent generations with high efficiency. Our method underlines the importance of taking each species' unique biology into account for the successful application of genome editing methods to non-traditional model organisms. We predict that these genome editing protocols will help in implementing $T$. domestica as a laboratory model and to further accelerate the development and application of useful genetic tools in this species.

\section{Introduction}

Thermobia domestica belongs to one of the most basal insect orders, Zygentoma, which retains an ancestral ametabolous and wingless life cycle. Such basal phylogenetic position and ancestral characteristics set this species as an attractive model for studying the mechanisms underlying the success of insects on Earth, which cover over $70 \%$ of the described animal species ${ }^{1}$. T. domestica has long been used mainly to study ancestral characteristics of insect physiology because of its suitable features as a laboratory model, such as a relatively short lifecycle (2.5-3.0 months from embryo to reproductive adult; Figure 1A) and an easy breeding. In the past three decades, its use has been expanded to investigate ancestral characteristics of various traits such as body plan, neural differentiation, and circadian rhythms ${ }^{2,3,4}$.

The application of advanced genetic tools in T. domestica could further accelerate such contributions in a wide research area. Successful RNA interference (RNAi)-mediated gene knockdown in embryos, nymphs, and adults has been reported in $T$. domestica ${ }^{4,5,6}$. The efficiency of systemic RNAi is still highly species-dependent-for example, it is 
generally high in coleoptera whereas it is low in the lepidoptera order ${ }^{7}$. The efficiency and duration of the RNAi knockdown in $T$. domestica is yet to be assessed. In addition to $\mathrm{RNAi}$, we have previously reported a successful CRISPR/Cas9-mediated gene knockout in $T$. domestica $^{8}$. The CRISPR/Cas system has been widely applied for genome editing in insects particularly for targeted gene knockout. Its use could be expanded for other applications such as gene reporter assay, cell lineage tracking, and manipulation of transcriptional activity by knocking-in exogenous constructs after the establishment of a protocol for delivering components of the CRISPR/Cas system into nuclei ${ }^{9}$. Combined with the published genome assembly $^{10}$, the wide use and further development of the CRISPR/Cas-based genome editing in T. domestica would facilitate studies focusing on the evolutionary mechanisms behind the outstanding adaptive success of insects. Here, we describe a detailed protocol for embryo microinjection and for mating adult $T$. domestica to generate a mutant strain using CRISPR/Cas9. Considering this novel method, we discuss the importance of considering the unique biology of nontraditional model species for successful applications of these techniques.

\section{Protocol}

\section{Maintenance of laboratory colonies}

1. For the maintenance of wildtype and mutant populations, use a large plastic container $(460 \mathrm{~mm} \times 360 \mathrm{~mm} \times 170$ $\mathrm{mm}$ ) with regular artificial fish food, water in plastic cups with a ventilation hole on the top, a folded paper for hiding the insects, and layered cotton for laying eggs (Figure 2A). Keep all T. domestica cultures inside $37^{\circ} \mathrm{C}$ incubators and set the relative humidity $(\mathrm{RH})$ inside each container to $60 \%-80 \%$.

NOTE: Because T. domestica absorbs water vapor from the atmosphere ${ }^{11}$, a direct water supply is not needed. The appropriate $\mathrm{RH}$ is maintained due to the presence of water vapor from plastic cups containing a ventilation hole on the top or from lidless cups inside each container. There is no need to humidify inside an entire incubator which contains cultures. The approximate duration of each developmental stage under the condition described in this protocol is shown in Figure 1. The developmental speed could be adjusted by changing the temperature and/or $\mathrm{RH}^{12}$.

2. Add food periodically. Add water before it dries up.

3. Transfer the populations to a new clean container at least every 3 months given that adults stop laying eggs in a dirty environment and/or dense population (see Discussion).

\section{Egg collection and microinjection}

1. Design a guide RNA (gRNA)

1. Design a gRNA sequence for each required target and BLAST the gRNA sequences against the genome assembly to check possible off-target recognition sites.

2. Synthesize and purify the gRNAs according to the manufacturer's instructions ${ }^{8}$.

NOTE: As an example, in the case of targeting the ATP-binding cassette transporter white gene, a 20 bp target sequence was designed and two synthesized DNA oligonucleotides 5'TAATACGACTCACTATAGTAAGTGTTGTGGGAC-3 and $5^{\prime}-$ 
' were ordered. The DNA template was prepared by annealing these two oligonucleotides followed by PCR amplification and the gRNA is then in vitro transcribed from the template with a T7 RNA polymerase.

\section{Prepare egg collection colonies}

1. Transfer about 20 male and 20 female adults to a middle-sized container (200 mm x $150 \mathrm{~mm}$ x $90 \mathrm{~mm}$ ) with food, water supply, a folded paper, and a small piece of layered cotton for egg laying (Figure 2B).

2. Set up several colonies to obtain large number of staged embryos in a short time period to be used for genome editing.

NOTE: About 20-40 eggs are expected to be collected from a colony after $8 \mathrm{~h}$ at $37^{\circ} \mathrm{C}$. It usually takes a few days for transferred adults to start laying eggs, possibly due to adaptation to a new environment.

3. On the day of injection, replace the cotton inside the containers with new ones.

4. Place a $76 \mathrm{~mm} \times 5 \mathrm{~mm}$ double-sided tape on a regular 76 $\mathrm{mm} \times 26 \mathrm{~mm}$ glass slide.

5. Eight hours later, collect the eggs from the layered cotton by separating the layers using forceps.

6. Align the eggs on the double-sided tape using a wet paint brush and keep a $2 \mathrm{~mm}$ distance between the eggs. All eggs should be oriented so that the longitudinal axis of an egg faces the injection side (Figure 3A). Gently press down the eggs with a paint brush for firm holding during the injection.

NOTE: In this protocol, fungus grows fast in damaged injected eggs under wet and warm condition. It is important to keep the distance between the eggs to prevent cross contamination and expansion of fungus.

7. Mix the gRNA and Cas9 protein to a final concentration of $100 \mathrm{ng} / \mu \mathrm{L}$ and $500 \mathrm{ng} / \mu \mathrm{L}$, respectively. Use distilled water for dilution. Incubate the $\mathrm{mix}$ for $10 \mathrm{~min}$ at room temperature to promote ribonucleoprotein complex formation and then keep the mix on ice.

NOTE: Neutral red at a $1 \%$ final concentration could be added to the injection solution to monitor the injected amount.

8. Load $2 \mu \mathrm{L}$ of the gRNA/Cas9 solution in a glass injection capillary with a microloader. Make sure there are no air bubbles in the solution before the injection. If necessary, tap the needle to remove the bubbles.

NOTE: In this experiment, a ready-made needle is used to obtain a fine needle tip (Figure 3B). A homemade needle with a similar shape could be used instead.

9. Fix the glass injection capillary, previously loaded with the gRNA/Cas9 solution, to a holder equipped with a manipulator and connect the holder to an electronic microinjector.

10. Optimize the shape of the needle tip by breaking it slightly with forceps to prevent clogging and getting better durability throughout a series of injections (an example of an appropriate needle is shown in Figure $\mathbf{3 C}$ ).

NOTE: It is recommended to change a needle when it is clogged. It is possible to continue injecting with the same needle if it is broken again with forceps, but a wider tip leads to more egg damage and lowers their survival rate. As $T$. domestica eggs are soft and fragile, keeping a fine needle tip is key for having a high survival rate after injection. 
11. Insert the needle at the midpoint of the longitudinal axis of an egg and inject a slight amount of the solution (see Figure 3D-H for reference on the amount of injection). Adjust the configuration of the electronic microinjector during injection, depending on the shape of the needle tip. NOTE: It is recommended to apply a constant pressure during injection, otherwise sticky egg contents can easily flow into the glass needle and may clog it. The solution may be to either inject with a short pressure pulse or with a constant pressure, depending on the amount of liquid injected. Keep in mind that when a needle tip has a wide opening, too much solution is injected into an egg, which causes lethality (Figure $\mathbf{3 F}-\mathbf{H}$ ). In that case, maintain a constant liquid flow by constant pressure, then insert the needle into an egg and pull it out immediately. If it causes too much overflow or the egg bursts, change the needle.

12. Keep the injected eggs in a container with the appropriate size for the number of injected eggs (<20 eggs: small dish; $>20$ eggs: middle-sized container) with $60 \%-80 \% \mathrm{RH}$ and $37^{\circ} \mathrm{C}$.

\section{Mating}

1. Check the injected eggs periodically and discard damaged eggs with forceps to avoid fungal growth (Figure $3 \mathbf{l}, \mathbf{J}$ ). In case too much fungus is growing on an egg, clean up the surface of the egg with $70 \% \mathrm{EtOH}$.

2. Before hatching (approximately 10 days at $37^{\circ} \mathrm{C}$ after egg laying), dip the glass slide with the injected eggs into talcum powder to coat the surface of the double-sided tape. This will avoid the stacking of hatched nymphs. Transfer the powder-coated glass slides to a middle-sized container with food, water, and a folded paper for hiding of the insects.
3. Remove the glass slides after the nymphs have hatched. Periodically supply food until they reach adulthood.

NOTE: It takes about 2.0-2.5 months for individuals to reach adulthood after they hatch (Figure 1A). The adult stage is judged based on a well-developed ovipositor in females (Figure 1B).

4. To mate the individuals, transfer as many as needed wildtype female adults from a laboratory colony to the medium-sized container and incubate them for at least 14 days at $37^{\circ} \mathrm{C}$ to make sure they are virgin.

NOTE: It is not necessary to collect virgin females from a laboratory colony because adult $T$. domestica have a repeated cycle of molting and mating called "reproductive and molting cycle", during which females throw away sperm with each molt and mate again in the next fertilization cycle ${ }^{13}$.

5. Transfer either a male or a female $\mathrm{G} 0$ adult that developed from an injected egg and wildtype adult(s) to a small plastic dish (Ø $100 \mathrm{~mm} \times 40 \mathrm{~mm}$ ) with food, a folded paper, and a small piece of cotton for laying G1 eggs (Figure 2C'; mating dish). Keep the mating dishes in a larger container with 60\%-80\% RH (Figure 2C).

NOTE: Multiple wildtype adults can be included in a dish to increase the chance of successful mating, although high success rates have been achieved with one-to-one pairing.

\section{Genotyping}

1. Design PCR primer pairs for each gRNA to amplify a 100-200 bp product that includes the site targeted by the gRNA. BLAST each primer sequence against the genome assembly to check its specificity (an example is shown for the targeting of the white gene in Figure 4A). 
2. Check the germline transformation of $\mathrm{G} 0$ adults.

1. Five days after the eggs are laid, collect individual $\mathrm{G} 1$ eggs from each $\mathrm{G} 0$ adult pair into $0.2 \mathrm{~mL}$ tubes (one egg per tube; store collected samples at $-20^{\circ} \mathrm{C}$ for a long-term storage). Separate the cotton layers to collect the eggs.

NOTE: It is recommended to genotype at least 12 G1 nymphs from each mating pair to evaluate the success of the germline transmission (see Representative Results).

2. Add $15 \mu \mathrm{L}$ of a $0.25 \mathrm{mg} / \mathrm{mL}$ Proteinase $\mathrm{K}$ solution (dissolved in Tris-EDTA buffer) to each tube, briefly homogenize samples with toothpicks, and incubate at $55^{\circ} \mathrm{C}$ for 3 to $16 \mathrm{~h}$.

3. Inactivate the Proteinase $\mathrm{K}$ by placing the samples at $95{ }^{\circ} \mathrm{C}$ for $10 \mathrm{~min}$.

4. Add $90 \mu \mathrm{L}$ of distilled water to each tube and mix well. Use $2 \mu \mathrm{L}$ of supernatant in a $10 \mu \mathrm{L}$ PCR reaction mix containing the primers designed in step 4.1.

NOTE: The use of a DNA polymerase optimized for crude templates is recommended to reach enough PCR amplification.

5. To analyze the PCR products, perform an heteroduplex mobility assay (HMA) with a microchip electrophoresis system (Figure 3B; see Ohde et al., 2018) $)^{8}$

NOTE: Mutations could be assessed with two alternative methods: (1) HMA with standard gel polymers, such as $8 \%$ polyacrylamide ${ }^{14}$; (2) digestion of PCR products with T7 endonuclease followed by agarose gel electrophoresis ${ }^{15}$.
6. Keep only the $\mathrm{G} 1$ nymphs resulting from $\mathrm{G} 0$ adults that contain mutations in their germline and discard the others.

3. Individual genotyping of G1 nymphs/adults

1. Isolate $\mathrm{G} 1$ nymphs into 24-well plates with an aspirator or a paint brush. Place the 24-well plates in a larger container (e.g., the medium-sized container used in this protocol) with water supply as described above to keep a $\mathrm{RH}$ of $60 \%-80 \%$ (Figure 1D). Maintain the supply of artificial regular fish food (Figure 1D').

NOTE: Although this step can be performed at any point of nymphal and adult stages, it is recommended to perform it after reaching adulthood and just before pairing (>2.5 months after injection; Figure 1B) because it is easier to maintain firebrats in a large container. Individual rearing is required to track the genotype of each $\mathrm{G} 1$ nymph on the following steps. $\mathrm{G} 1$ nymphs from the same $\mathrm{G} 0$ adult can have different mutations.

2. Pinch and pull cerci and the caudal filament from a nymph/adult using forceps and collect them into a 0.2 $\mathrm{mL}$ tube containing $50 \mu \mathrm{L} \mathrm{EtOH}$ (store the collected samples at $-20^{\circ} \mathrm{C}$ for a long-term storage).

NOTE: Tissue samples are collected in $\mathrm{EtOH}$ because it prevents the loss of these small samples due to static electricity. If one needs to stop the motion of insects, anesthetize nymph/adult on ice when taking tissue samples. Because $T$. domestica cannot survive after long-term cooling on ice, do not anesthetize them for more than a minute and immediately move them back to room temperature. 
Ablation of cerci and the caudal filament causes no increase of mortality.

3. Place sample tubes with the lids open on a thermal block for $15 \mathrm{~min}$ at $70{ }^{\circ} \mathrm{C}$ to evaporate the $\mathrm{EtOH}$.

4. Repeat steps 4.2.2-4.2.5 for genotyping.

5. Submit the PCR products in which a mutant band pattern is observed to a standard Sanger sequencing service.

6. Keep the G1 nymphs/adults with the desired mutations and discard the others (see Figure $4 \mathrm{C}$ for a representative sequencing result).

4. Cross the adults containing the desired mutations in a mating dish and obtain the next generations to establish a homozygous mutant strain.

\section{Representative Results}

In our hands, about 100 eggs can be well injected with a single injection capillary when it has the adequate tip (Figure 3C). Injection of gRNA/Cas9 ribonucleoprotein complex in embryos within the first $8 \mathrm{~h}$ after egg laying results in indels at the gRNA targeted site. This causes biallelic mutations in some cells of the injected generation (G0) and thus mutant mosaic phenotypes are usually obtained in G0. For example, when this protocol was used to inject a gRNA that is designed to target the white gene, $32.6 \%$ of G0 nymphs display partial loss of pigmentation in their compound eyes and dorsal regions (Figure 5$)^{8}$.

Using the presently described dry injection method, when $80-120$ eggs are injected the survival rate of the injected embryos is as high as $40 \%-60 \%$. This is in contrast with the previous wet injection method, in which eggs are injected and maintained on an agarose plate, occasionally resulting in a survival rate of less than $10 \%$.

Assessment of the germline transformation of $\mathrm{G} 0$ adults and mutated G1 individuals was done by genomic PCR followed by HMA. In HMA, wildtype and mutant alleles anneal in each possible combination, which typically results in four distinct bands on a gel electrophoresis (two homoduplexes and two heteroduplexes) ${ }^{14}$. In G1 samples, differential band patterns between wildtype and mutated samples are clearly distinguishable (Figure 4B). Germline transformation was found in $39.1 \%$ of the G0 adults when we targeted the white gene ${ }^{8}$. In our experience, the percentage of mutated individuals in $\mathrm{G} 1$ nymphs from a single $\mathrm{G} 0$ pair varies from $25 \%$ to $100 \%$.

To evaluate the effect of our mating environment on the mating success, we crossed wildtype adults in a mating dish and obtained a success rate of $95.8 \%$ (23/24 pairs). 


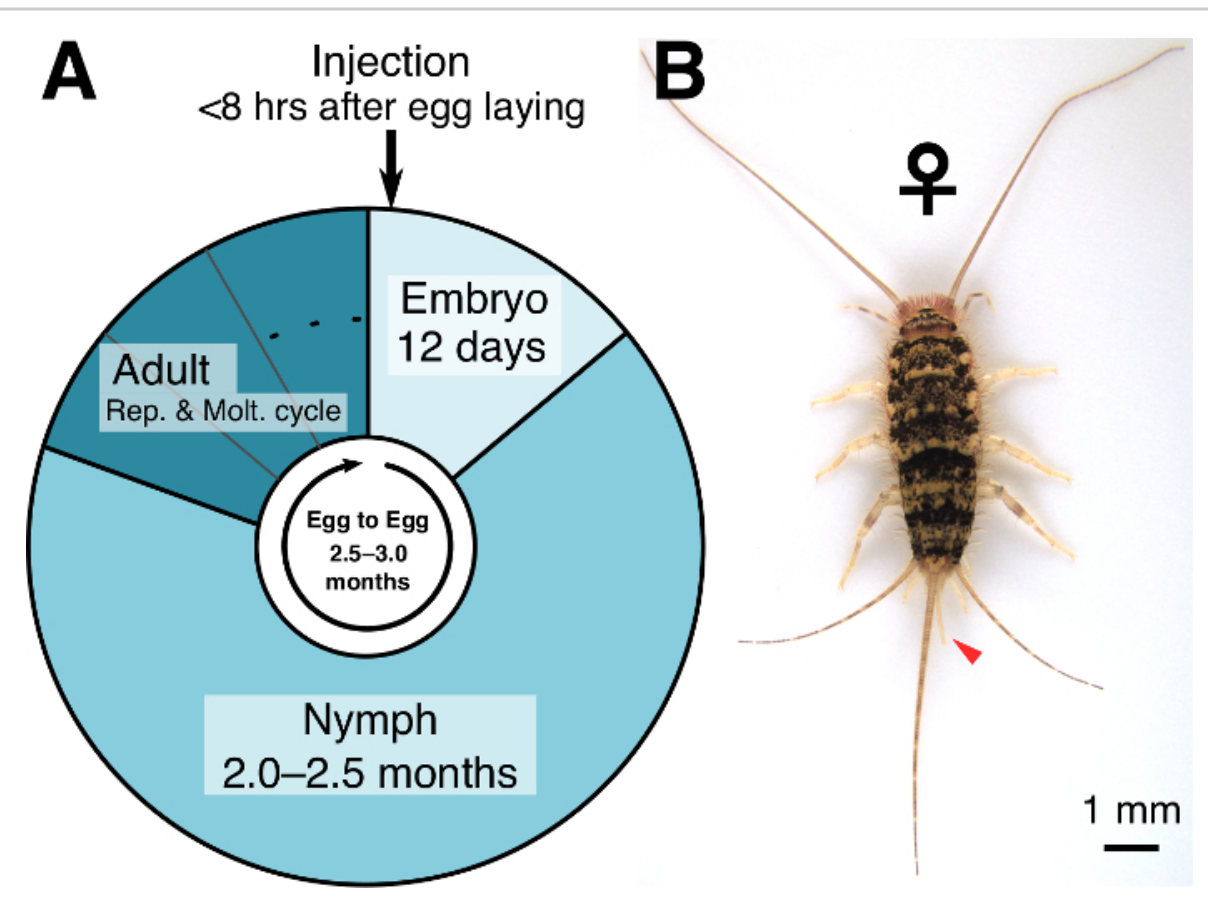

Figure 1: The lifecycle of $\boldsymbol{T}$. domestica. (A) Approximate duration of each developmental stage in T. domestica. (B) Dorsal view of an adult female. Arrowhead indicates a well-developed ovipositor. Please click here to view a larger version of this figure. 

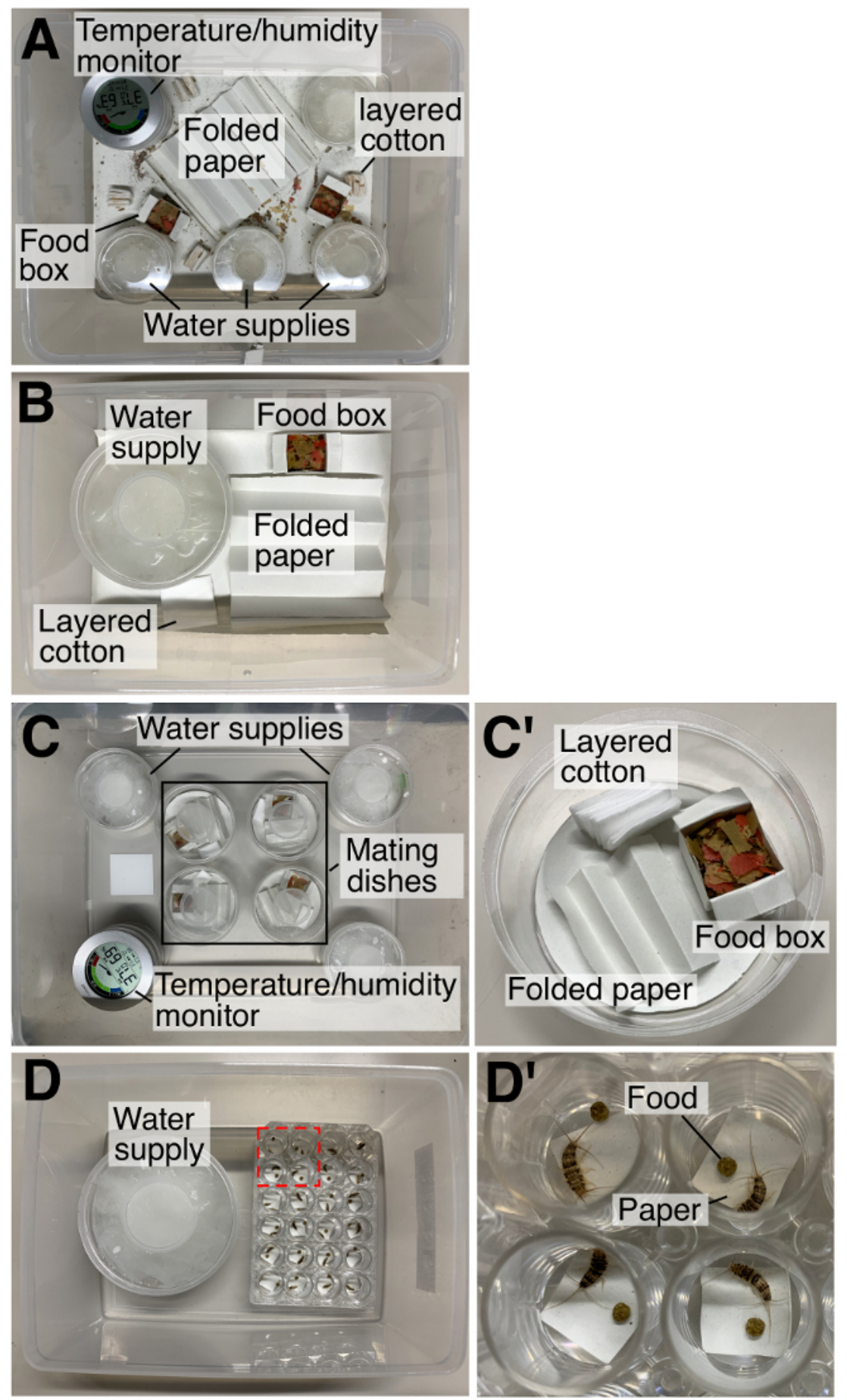

Figure 2: Artificial environments used in this protocol. (A) A large container for laboratory colony, (B) a medium-size container for egg collection, (C) mating dishes with water supplies in a large container, (C') a mating dish, (D) a 24-well plate with water supply in the medium-size container. Boxed region is magnified in (D') to show a T. domestica individual. Please click here to view a larger version of this figure. 

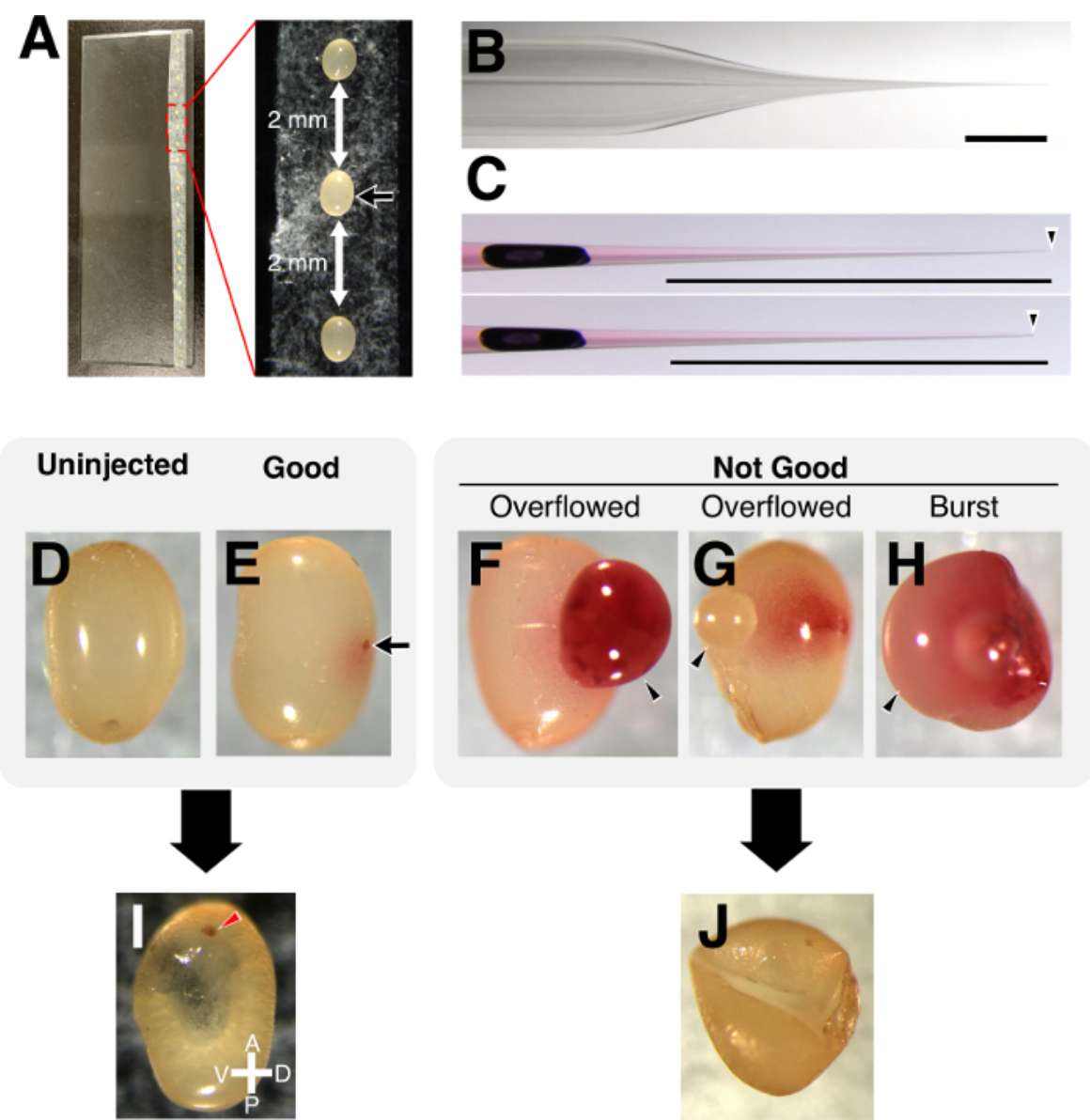

Figure 3: Dry injection of $\boldsymbol{T}$. domestica eggs. (A) Eggs aligned on a glass slide. Black arrow indicates the point where a needle is inserted. (B) Shape of a glass needle tip used for injection. (C) The same glass needle before (top) and after (bottom) breaking the tip. Needles were filled with $1 \%$ neutral red. Arrowhead indicates the tip of the needle. Scale bar is $1 \mathrm{~mm}$. (D) Uninjected egg. (E) A good example of an injection. Arrow indicates the point of injection. (F-H) The solution is overflowing from the injected site $(\mathbf{F})$ or from the opposite side $(\mathbf{G})$ of the injected egg; too much volume of injection caused a burst (H). Arrowhead indicates overflowed egg content. (I) Normally developed late embryo. Arrowhead indicates the colored compound eye. ( $\mathbf{J})$ Shrunken damaged egg 3 days after injection. Please click here to view a larger version of this figure. 


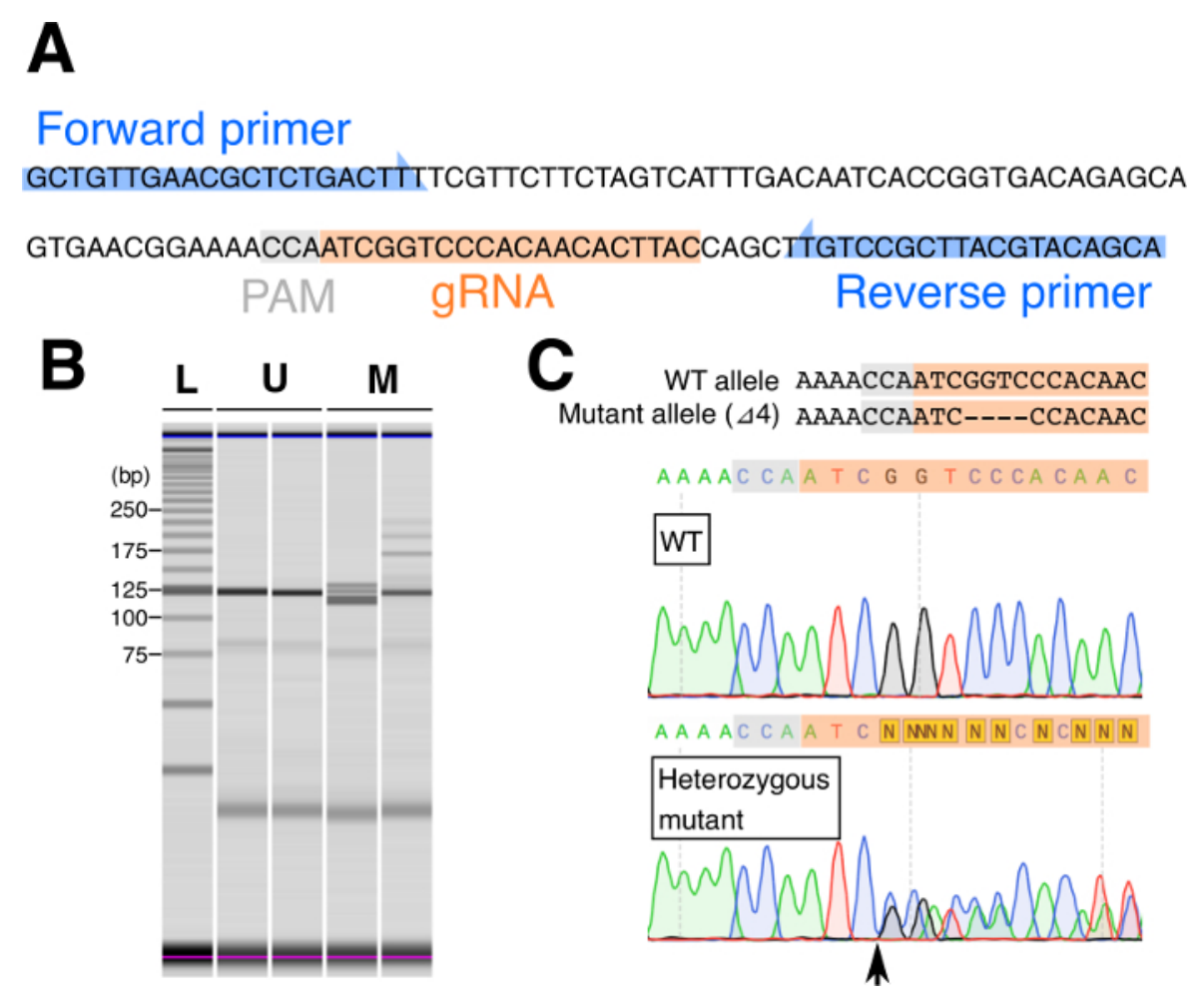

Figure 4: Genotyping of G1 individuals. (A) PCR primers are designed to amplify a 120 bp genomic region that includes the gRNA target site. (B) Multiple bands of homoduplex and heteroduplex DNAs are detected in mutated samples while single bands appear in unmutated samples. L: DNA ladder, U: unmutated samples, M: mutated samples. (C) Representative result of direct sequencing of PCR products from wildtype and heterozygous mutant samples. Sequences of wildtype and mutant $(\Delta 4)$ allele are shown on top. The forward primer shown in $(A)$ was used as a sequencing primer. The sequence of a heterozygous mutant (bottom) is indicated by two overlapping sequences from the predicted cleavage site (arrow). Please click here to view a larger version of this figure. 


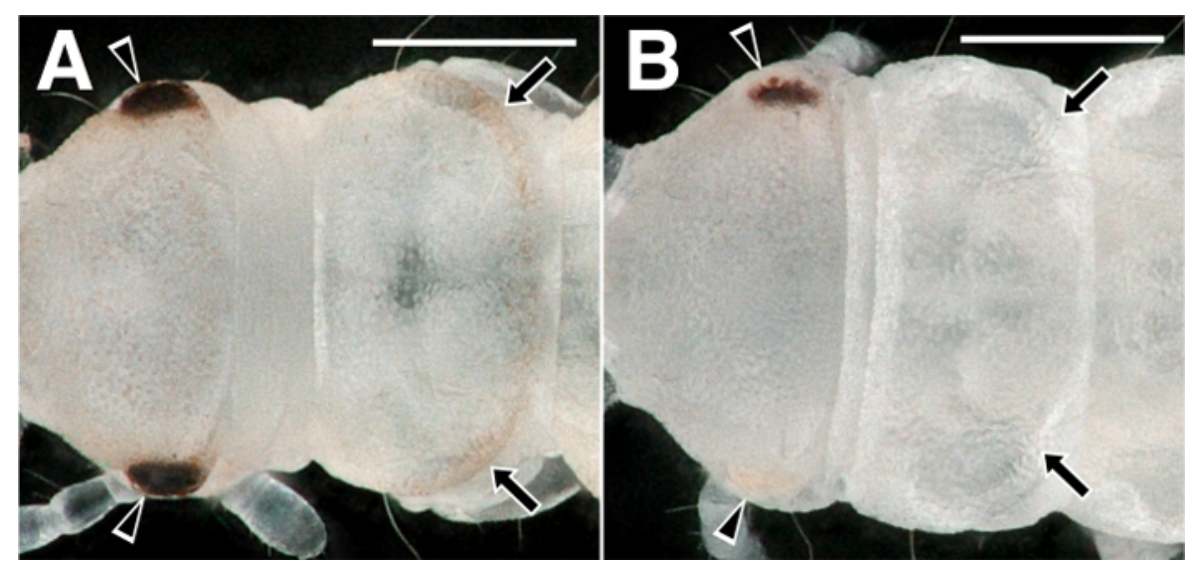

Figure 5: Mosaic loss of pigmentations in compound eyes and in the dorsal region after targeting the white gene with gRNA/Cas9 protein injection. (A) Wildtype and (B) white gRNA/Cas9 protein-injected first instar nymphs. Partial loss of black and pink pigmentations in eyes (arrowhead) and in the dorsal region (arrow) are indicated. Scale bar is $200 \mu \mathrm{m}$. Please click here to view a larger version of this figure.

\section{Discussion}

For the successful generation of the desired T. domestica mutant with CRISPR/Cas9, it is first important to collect a sufficient number of staged embryos for injection. For a constant collection of a sufficient number of $T$. domestica eggs, the key is to select an appropriate size of the container to have a lower population density because it would help the successful completion of a series of complex mating behaviors, which is repeated after every adult molt ${ }^{13}$. A male T. domestica adult transfers its sperm indirectly to a female via a spermatophore. Sweetman (1938) reported that it takes male adults 20 to $35 \mathrm{~min}$ from the initiation of the mating behavior to the placement of a spermatophore ${ }^{12}$. It is likely that disturbance of the interaction between a mating pair by other individuals prevents successful fertilization, which could happen more frequently in a dense environment.

Although eggs are collected $8 \mathrm{~h}$ after replacing the cotton inside a container to collect enough eggs in our protocol, the higher efficiency of genome editing may be achieved by collecting eggs and injecting them within a shorter time (e.g., 4 $\mathrm{h}$ after egg laying) when injected materials have more chance to be delivered to the large proportion of nuclei. Injection to a sufficient number of eggs within a shorter time could be done by (1) increasing the number or the size of containers if space allows, or (2) repeating the same procedure to obtain a sufficient number of injected eggs.

The site of injection is generally considered to be important for successful germline transformation in insects. T. domestica eggs are usually ellipsoidal in shape and contain a germ band at one pole of their longitudinal axis. It is recommended to inject the gRNA/Cas9 mixture at the midpoint of the egg longitudinal axis because it is hard to identify the pole where a germ band is formed in early embryos due to the variable shape of $T$. domestica egg. Although the gRNA/Cas9 solution is not directly injected to the site of the germ band formation, 
we have achieved germline transformation in as high as $39.1 \%$ of $\mathrm{GO}$ adults ${ }^{8}$.

During the microinjection of eggs, it is important to use a fine needle tip to obtain a high survival rate, as it is the case for other animal models. We obtained a higher survival rate after injection with ready-made needles than when using homemade glass needles in $T$. domestica eggs. However, a similarly high survival rate could be achieved by replicating the fine needle shape with homemade needles (as shown in Figure 3B,C). According to our experience, the dry injection method results in a higher survival rate than the previously reported wet injection method ${ }^{8}$. We found that egg incubation in a dry environment is key for this improvement, taking advantage of the fact that $T$. domestica eggs and early nymphs are resistant to desiccation. This is in accordance with previous reports suggesting that this species prefers a dry environment particularly during early developmental stages and that a wet environment can even be harmful ${ }^{16}$. Instead of a plastic plate, an agarose gel can be used for a quick alignment of the eggs; however, transferring the injected eggs to a dry surface leads to a higher survival rate.

In conclusion, our method underlines the importance of taking the unique biology of $T$. domestica into account for a successful genome editing: keeping a sparse environment for collecting a sufficient number of eggs and a dry environment for having a higher survival rate of injected embryos. The basic protocols for injection, mating, and culture maintenance reported here are used not only for generating mutant strains with genome editing, but also for applications of other genetic tools such as RNAi and transgenesis and will help to understand the mechanisms underlying the early evolution of insects.

\section{Disclosures}

The authors have nothing to disclose.

\section{Acknowledgments}

TO and TD were supported by JSPS KAKENHI grant numbers $19 \mathrm{H} 02970$ and $20 \mathrm{H} 02999$, respectively.

\section{References}

1. Roskov, Y. et al. eds. Species 2000, ITIS Catalogue of Life, 2019 Annual Checklist. http:// www.catalogueoflife.org/annual-checklist/2019. (2019).

2. Peterson, M. D., Rogers, B. T., Popadić, A., Kaufman, T. C. The embryonic expression pattern of labial, posterior homeotic complex genes and the teashirt homologue in an apterygote insect. Development Genes and Evolution. 209, 77-90 (1999).

3. Farris, S. M. Developmental organization of the mushroom bodies of Thermobia domestica (Zygentoma, Lepismatidae): Insights into mushroom body evolution from a basal insect. Evolution and Development. 7, 150-159 (2005).

4. Kamae, Y., Tanaka, F., Tomioka, K. Molecular cloning and functional analysis of the clock genes, Clock and cycle, in the firebrat Thermobia domestica. Journal of Insect Physiology. 56, 1291-1299 (2010).

5. Ohde, T., Masumoto, M., Yaginuma, T., Niimi, T. Embryonic RNAi analysis in the firebrat, Thermobia domestica: Distal-less is required to form caudal filament. Journal of Insect Biotechnology and Sericology. 105, 99-105 (2009).

6. Ohde, T., Yaginuma, T., Niimi, T. Nymphal RNAi analysis reveals novel function of scalloped in antenna, 
cercus and caudal filament formation in the firebrat, Thermobia domestica. Journal of Insect Biotechnology and Sericology. 108, 101-108 (2012).

7. Bellés, X. Beyond Drosophila: RNAi in vivo and functional genomics in insects. Annual Review of Entomology. 55, 111-128 (2010).

8. Ohde, T., Takehana, Y., Shiotsuki, T., Niimi, T. CRISPR/Cas9-based heritable targeted mutagenesis in Thermobia domestica: A genetic tool in an apterygote development model of wing evolution. Arthropod Structure and Development. 47, 362-369 (2018).

9. Nji, C. et al. CRISPR/Cas9 in insects: Applications, best practices and biosafety concerns. Journal of Insect Physiology. 98, 245-257 (2017).

10. Brand, P. et al. The origin of the odorant receptor gene family in insects. eLife. 7, 1-13 (2018).

11. Noble-Nesbitt, J. Water balance in the firebrat, Thermobia domestica (Packard). Exchanges of water with the atmosphere. Journal of Experimental Biology. 50, 745-769 (1969).

12. Sweetman, H. L. Physical ecology of the firebrat, Thermobia domestica (Packard). Ecological Monographs. 8, 285-311 (1938).

13. Nijhout, H. F. Insect Hormones. Princeton University Press. Princeton, NJ (1994).

14. Chen, J. et al. Efficient detection, quantification and enrichment of subtle allelic alterations. DNA Research. 19, 423-433 (2012).

15. Mashal, R. D., Koontz, J., Sklar, J. Detection of mutations by cleavage of DNA heteroduplexes with bacteriophage resolvases. Nature Genetics. 9, 177-183 (1995).
16. Adams, J. A. Biological notes upon the firebrat, Thermobia domestica Packard. Journal of the New York Entomological Society. 41, 557-562 (1933). 